\title{
The effect of chemical treatment on reduction of aflatoxins and ochratoxin $A$ in black and white pepper during washing.
}

\begin{abstract}
The effect of 18 different chemicals, which included acidic compounds (sulfuric acid, chloridric acid, phosphoric acid, benzoic acid, citric acid, acetic acid), alkaline compounds (ammonia, sodium bicarbonate, sodium hydroxide, potassium hydroxide, calcium hydroxide), salts (acetate ammonium, sodium bisulfite, sodium hydrosulfite, sodium chloride, sodium sulfate) and oxidising agents (hydrogen peroxide, sodium hypochlorite), on the reduction of aflatoxins B1, B2, G1 and G2 and ochratoxin A (OTA) was investigated in black and white pepper. OTA and aflatoxins were determined using HPLC after immunoaffinity column clean-up. Almost all of the applied chemicals showed a significant degree of reduction on mycotoxins $(\mathrm{p}<0.05)$. The lowest and highest reduction of aflatoxin $\mathrm{B} 1$, which is the most dangerous aflatoxin, was $20.5 \% \pm 2.7 \%$ using benzoic acid and $54.5 \% \pm 2.7 \%$ using sodium hydroxide. There was no significant difference between black and white peppers $(\mathrm{p}<0.05)$.
\end{abstract}

Keyword: High-performance liquid chromatography; Aflatoxins; Ochratoxin A; Plants. 